Archive for

Organic Chemistry

Arkivoc 2017, part ii, 546-556

\title{
Synthesis of new alkylated and methoxylated analogues of ebselen with antiviral and antimicrobial properties
}

Magdalena Piętka-Ottlik, ${ }^{* a}$ Małgorzata Burda-Grabowska, ${ }^{b}$ Marta Woźna, ${ }^{a}$ Joanna Waleńska, ${ }^{\text {a }}$ Rafał Kaleta, ${ }^{b}$ Ewa Zaczyńska, ${ }^{\mathrm{C}}$ Egbert Piasecki, ${ }^{\mathrm{c}}$ and Mirosław Giurg ${ }^{\mathrm{b}}$

${ }^{a}$ Wrocław University of Science and Technology, Department of Organic and Pharmaceutical Technology,

Wybrzeże Wyspiańskiego 27, 50-370 Wrocław, Poland,

${ }^{b}$ Wrocław University of Science and Technology, Department of Organic Chemistry, Wybrzeże Wyspiańskiego

27, 50-370 Wrocław, Poland,

'Hirszfeld Institute of Immunology and Experimental Therapy, Polish Academy of Sciences, Weigla 12, 53-114

Wrocław, Poland

E-mail: magdalena.pietka@pwr.edu.pl

Dedicated to Prof. Jacek Młochowski on the occasion of his $80^{\text {th }}$ anniversary

Received 07-13-2016 Accepted 09-20-2016 Published on line 02-06-2017

\section{Abstract}

A series of new mono and disubstituted alkylated and methoxylated benzisoselanzol-3(2H)-ones and bis(2carbamoylaryl)diselenides were prepared in yields ranging from 55\% to $95 \%$ starting from anthranilic acid and were evaluated for antiviral and antimicrobial activity. The compounds exhibited antiviral activity against Human herpes virus 1 and Encephalomyocarditis virus as well as antimicrobial activity against Staphylococcus aureus, Bacillus subtilis, Escherichia coli and Candida albicans.<smiles>NC(=O)c1ccccc1S</smiles><smiles></smiles><smiles>CC#CC</smiles><smiles>Nc1ccccc1C(=O)O</smiles><smiles>C1CC2CC1C2</smiles><smiles>O=C(Nc1cc[R]cc1)c1ccccc1S(=O)(=O)O</smiles>

$\mathrm{R}^{1}, \mathrm{R}^{2}=\mathrm{Me}, \mathrm{OMe}, \mathrm{Cl}, t-\mathrm{Bu}, \mathrm{H}$

Keywords: Ebselen, organoselenium compounds, benzisoselenazol-3(2H)-ones, diselenides, antiviral activity, antimicrobial activity 


\section{Introduction}

Over last few decades organoselenium compounds have been extensively studied in organic and medicinal chemistry. ${ }^{1-15}$ Due to their unique properties, they attracted a wide interest as reagents in new synthetic routes, ${ }^{3}$ promising catalysts for various oxidation reactions ${ }^{1-7,16-19}$ and potential pharmaceuticals..$^{8-15,20,21}$ Ebselen (2-phenylbenzisoselenazol-3(2H)-one) and its analogues have received particular attention as glutathione peroxidase mimics, ${ }^{8,11,15}$ antioxidant and anti-inflammatory agents. ${ }^{13-15}$ Furthermore, the continuing interest in this class of compounds has led to the development of various structures with antiviral ${ }^{22-26}$ and antimicrobia ${ }^{24-27}$ properties. In this work we report a convenient synthetic route to a series of new alkylated and methoxylated benzisoselanazol-3(2H)-ones $\mathbf{4}$ and bis(2-carbamoylaryl)diselenides $\mathbf{6}$ which were tested for antiviral and antimicrobial activity in biological studies.

\section{Results and Discussion}

Searching for new antiviral and antimicrobial agents, we report here the synthesis of new alkylated and methoxylated analogues of ebselen - an organoselenium compound well known for its variety of biological and oxygen transfer properties. 2-Phenylbenzisoselanazol-3(2H)-ones alkylated and methoxylated on the benzene ring $4 \mathrm{a}-\mathrm{I}$ were prepared through a tandem selenenylation-acylation of mono and disubstituted anilines with 2-(chloroseleno)benzoyl chloride (3), in the presence of triethylamine as a base, whereas

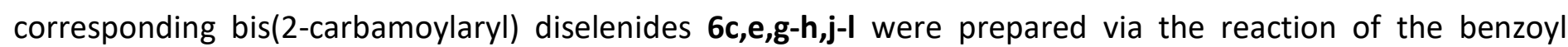
chloride 5 with appropriate anilines in the presence of sodium carbonate (Scheme 1). Both benzoyl chlorides 3 and $\mathbf{5}$ were obtained in a three-step synthesis starting from anthranilic acid (1), which was diazotized and reacted with dilithium diselenide in aprotic medium (THF) to give after acidification 2,2'-diselenobisbenzoic acid $\mathbf{2}$ with $78 \%$ yield. ${ }^{28-30}$ Efficient cooling is important to reduce by-product formation such as salicylic acid. Dilithium diselenide was generated in situ from elemental lithium and selenium in the presence of 4,4'-di(tbutyl)biphenyl, as a catalyst. ${ }^{31}$ Alternatively, disodium diselenide in protic medium (MeOH) can be used to introduce the diselenide moiety, however the yield was reported to be lower (18-67\%). 24,32 The procedure for preparation of disodium diselenide is critical to obtain 2,2'-diselenobisbenzoic acid (2) with good yields. The type of reducing agent, such as hydrazine monohydrate, ${ }^{24}$ and sodium tetrahydroborate ${ }^{21}$ as well as reaction times up to 48 hours, are of particular importance. Some authors report yields even up to $95 \%$ however no synthetic details were provided. ${ }^{20}$

The acid $\mathbf{2}$ was easily converted into 2-(chloroseleno)benzoyl chloride (3) (85\%) with thionyl chloride, used in excess, in the presence of catalytic amounts of $N, N^{\prime}$-dimethylformamide. ${ }^{29}$ When thionyl chloride was used in excess (2.5 equivalents), chloride 5 (64\%) was produced. ${ }^{29}$ The reaction of benzoyl chlorides $\mathbf{3}$ and $\mathbf{5}$ with anilines substituted at ortho, meta or para positions of the phenyl ring by electron-donating methyl and/or strong electron-donating methoxy groups, (in some cases with a chlorine substituent) provides a straightforward way to obtain new mono and disubstituted 2-phenylbenzisoselanzol-3(2H)-ones 4 and bis(2carbamoylaryl) diselenides 6 , respectively. All products were prepared with good to excellent yields (Scheme 1). An ortho effect of methyl and methoxy groups, and a steric hindrance effect of the tert-butyl substituent in the case of formation of compound $\mathbf{4 f}$, were not observed, only in the cases of selenazolones $\mathbf{4 d}$, and $\mathbf{4} \mathbf{j}$ were minor effects of electron-withdrawing chlorine substituent observed. This confirms that the general approach presented here can be successfully used for preparation of new substitued analogues of ebselen. To our best 
knowledge compounds $4 \mathbf{a}-\mathbf{h}, \mathbf{4 j}, \mathbf{6 c}, \mathbf{6 e}, \mathbf{6 g}, \mathbf{6 h}, \mathbf{6 j}, \mathbf{6 k}$ are new structures and were not previously described in the literature. All compounds were fully characterized by spectroscopic methods (see Experimental Section).

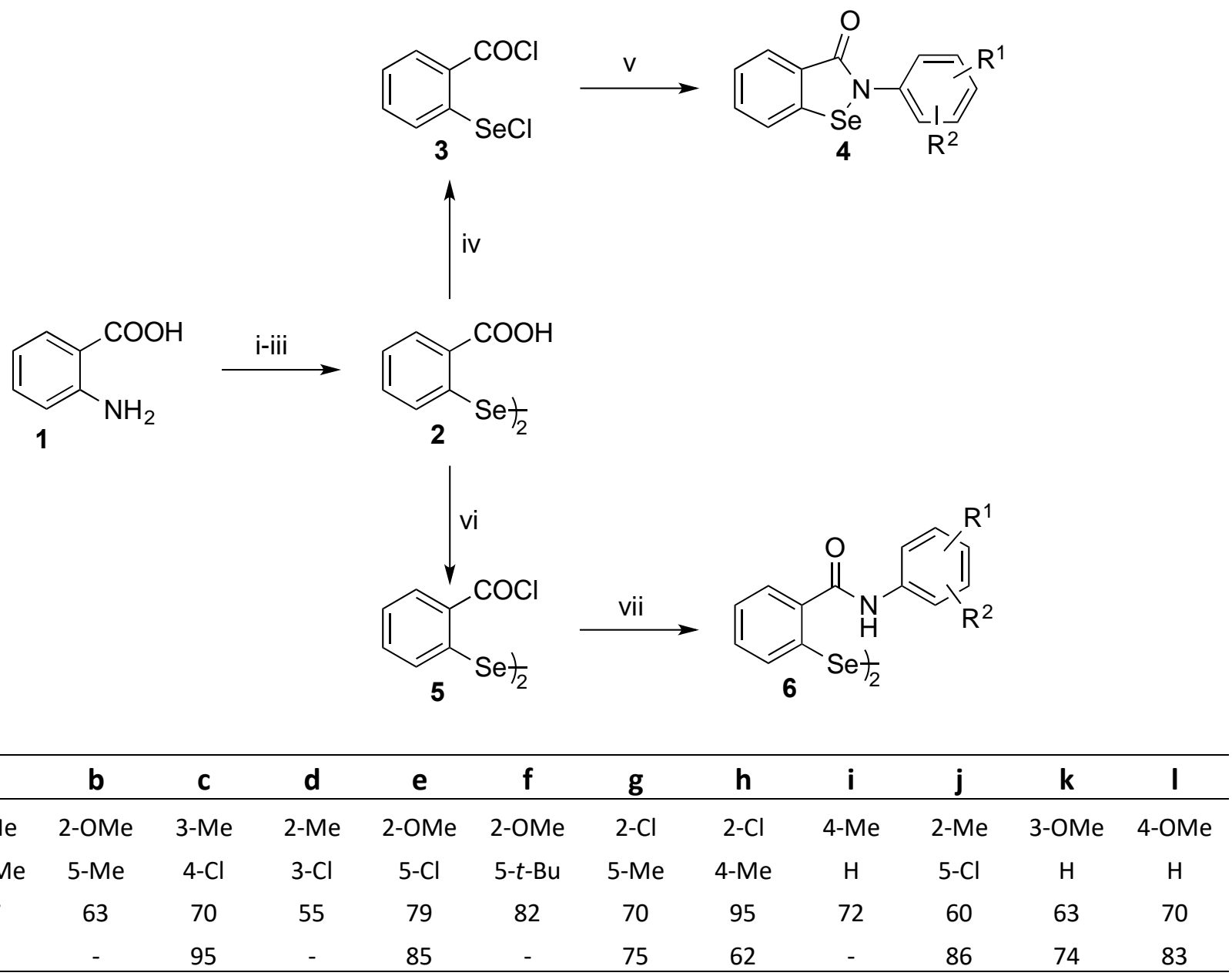

Reagents: i: $\mathrm{NaNO}_{2}, \mathrm{HCl},-7-5^{\circ} \mathrm{C}$, ii: $\mathrm{Li}_{2} \mathrm{Se}_{2}, \mathrm{NaOH}_{\mathrm{aq}},-7-0^{\circ} \mathrm{C}$, iii: $\mathrm{HCl}$, iv: $\mathrm{SOCl}_{2}$ (excess), DMF (cat.), $\Delta, \mathrm{v}^{\mathrm{R}} \mathrm{R}^{1} \mathrm{R}^{2}$ $\mathrm{C}_{6} \mathrm{H}_{3}-\mathrm{NH}_{2}, \mathrm{MeCN}, \mathrm{Et}_{3} \mathrm{~N}$, vi: 3,5 eq. SOCl , DMF (cat.), benzene, $\Delta$, vii: $\mathrm{R}^{1} \mathrm{R}^{2}-\mathrm{C}_{6} \mathrm{H}_{3}-\mathrm{NH}_{2}, \mathrm{CH}_{2} \mathrm{Cl}_{2}, \mathrm{Na}_{2} \mathrm{CO}_{3}$.

Scheme 1. Synthesis of alkylated and methoxylated benzisoselenazol-3(2H)-ones 4 and bis(2-carbamoylaryl)diselenides 6 .

\section{Antiviral and antimicrobial evaluation of organoselenium compounds}

The alkylated and methoxylated analogues of ebselen $\mathbf{4}$ and $\mathbf{6}$ were evaluated for antiviral activity against Human herpes virus 1 (HHV-1, Herpesviridae, enveloped virus), Encephalomyocarditis virus (EMCV, Picornaviridae, non-enveloped virus) and Vesicular stomatitis virus (VSV, Rhabdoviridae, enveloped virus) as well as antimicrobial activity against Staphylococcus aureus, Bacillus subtilis, Escherichia coli and Candida albicans by serial dilution techniques. ${ }^{26}$ Their activities were compared with those of ebselen.

The compounds exhibited high antiviral activity against HHV-1 and EMCV but in almost all cases were inactive towards VSV (Table 1). A similar tendency was observed in our previous studies. ${ }^{24-26}$ Generally, benzisoselenazol-3(2H)-ones 4 were more active than corresponding diselenides 6 . The results of screening of benzisoselenazol-3(2H)-ones 4 toward HHV-1 are particularly interesting. These compounds were more active than ebselen, with minimal inhibitory concentration (MIC) values in a range of $2-6 \mu \mathrm{g} / \mathrm{mL}$. The $\mathrm{most}$ 
promising as antiviral agents were compounds $\mathbf{4 b - d}$ and $\mathbf{6 c}$ with higher chemotherapeutic indices (I), indicating that the concentration active against virus was lower than that showing a cytotoxic effect.

Table 1. Antiviral activity and cytotoxicity of compounds 4 and 6

\begin{tabular}{cccccccc}
\hline Compound & $\mathrm{TCCD}_{50}{ }^{\mathrm{a}}$ & \multicolumn{2}{c}{ HHV-1 } & \multicolumn{2}{c}{ EMCV } & \multicolumn{2}{c}{ VSV } \\
\cline { 3 - 8 } & {$[\mu \mathrm{g} / \mathrm{mL}]$} & $\mathrm{MIC}^{\mathrm{b}}$ & $\mathrm{I}^{\mathrm{c}}$ & $\mathrm{MIC}^{\mathrm{b}}$ & $\mathrm{I}^{\mathrm{c}}$ & $\mathrm{MIC}^{\mathrm{b}}$ & $\mathrm{I}^{\mathrm{c}}$ \\
\hline $\mathbf{4 a}$ & 7.3 & 4 & 1.8 & 20 & 0.4 & n.a & - \\
$\mathbf{4 b}$ & 8.5 & 2 & 4.2 & 10 & 0.8 & n.a & - \\
$\mathbf{4 c}$ & 19.5 & 4 & 4.9 & 60 & 0.3 & n.a & - \\
$\mathbf{4 d}$ & 78 & 6 & 13 & $\mathrm{n} . \mathrm{a}$ & - & n.a & - \\
$\mathbf{4 e}$ & 8.5 & 4 & 2.1 & 20 & 0.4 & n.a & - \\
$\mathbf{4 f}$ & 4.9 & 6 & 0.8 & 8 & 0.6 & n.a & - \\
$\mathbf{4 g}$ & 11 & 4 & 2.7 & 8 & 1.4 & n.a & - \\
$\mathbf{4 h}$ & 7.3 & 4 & 1.8 & 10 & 0.7 & n.a & - \\
$\mathbf{4 i}$ & 3.7 & 2 & 1.8 & 40 & 0.09 & n.a & - \\
$\mathbf{4 j}$ & 8.5 & 4 & 2.1 & 10 & 0.9 & n.a & - \\
$\mathbf{6 c}$ & 58.5 & 10 & 5.8 & n.a & - & n.a & - \\
$\mathbf{6 e}$ & 22 & 40 & 0.5 & 200 & 0.1 & 400 & 0.06 \\
$\mathbf{6 g}$ & 58.5 & 20 & 2.9 & 60 & 1.0 & n.a & - \\
$\mathbf{6 h}$ & 58.5 & 100 & 0.6 & n.a & - & n.a & - \\
$\mathbf{6 j}$ & 19.5 & 200 & 0.1 & 800 & 0.02 & n.a & - \\
Ebselen & 12 & 8 & 1.5 & 10 & 1.2 & n.a & - \\
\hline
\end{tabular}

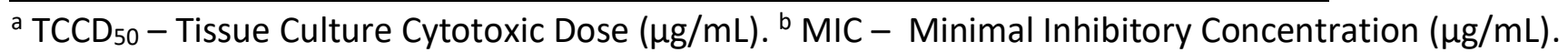
${ }^{c} \mathrm{I}=\mathrm{TCCD}_{50} / \mathrm{MIC}$. n.a - non-active, MIC $>1000 \mu \mathrm{g} / \mathrm{mL}$.

Table 2. Antimicrobial activity of compounds 4 and 6

\begin{tabular}{|c|c|c|c|c|c|}
\hline \multirow{2}{*}{ Compound } & \multicolumn{5}{|c|}{$\mathrm{MIC}^{\mathrm{a}}[\mu \mathrm{g} / \mathrm{mL}]$} \\
\hline & S. aureus & B. subtilis & E. coli & P. aeruginosa & C. albicans \\
\hline $4 a$ & 20 & 10 & 80 & n.a. & 80 \\
\hline $4 b$ & 20 & 20 & 80 & n.a. & 80 \\
\hline $4 e$ & 85 & 85 & 85 & n.a. & 42 \\
\hline $4 f$ & 33 & 33 & 33 & n.a. & 65 \\
\hline $4 k$ & 76 & 38 & 38 & n.a. & 68 \\
\hline 41 & 10 & 152 & 152 & n.a. & 76 \\
\hline $6 e$ & n.a & 340 & n.a & n.a. & n.a \\
\hline $6 k$ & 305 & n.a & n.a & n.a. & n.a \\
\hline 61 & n.a & n.a & n.a & n.a. & n.a \\
\hline Ebselen & 17 & 17 & 32 & n.a. & 128 \\
\hline
\end{tabular}

${ }^{a}$ MIC - Minimal Inhibitory Concentration ( $\left.\mu \mathrm{g} / \mathrm{mL}\right)$. n.a. - non-active, MIC > $512 \mu \mathrm{g} / \mathrm{mL}$.

Benzisoselenazol-3(2H)-ones 4 were found to be active against $S$. aureus, B. subtilis, E. coli and $C$. albicans (Table 2). None of compounds exhibited antibacterial activity against $P$. aeruginosa which is in agreement with previous observations. ${ }^{26}$ Usually this gram-negative bacterial strain is not sensitive to benzisoselenazol-3(2H)- 
ones and only a few structures were found to exhibit very low antibacterial activity towards it. ${ }^{24,26}$ Contrary to their cyclic analogues, diselenides appeared to be practically inactive against all tested microorganisms. Only 6e and $\mathbf{6 k}$ showed low activity against $B$. subtilis and $S$. aureus, respectively.

\section{Conclusions}

A series of new alkylated and methoxylated 2-phenylbenzisoselanzol-3(2H)-ones 4 and bis(2carbamoylaryl)diselenides 6 were prepared in good to excellent yields starting from anthranilic acid. This general approach proved to be convenient for the preparation of various mono- and disubstituted analogues of ebselen. The studies of antiviral and antimicrobial activity revealed new potential antiviral agents against HHV-1 and EMCV, and antimicrobial agents against Staphylococcus aureus, Bacillus subtilis, Escherichia coli and Candida albicans.

\section{Experimental Section}

General. Melting points were determined on an Electrothermal IA 91100 digital melting-point apparatus using the standard open capillary method. ${ }^{1} \mathrm{H}$ and ${ }^{13} \mathrm{C}$ NMR spectra were recorded in $\mathrm{CDCl}_{3}$ or DMSO- $d_{6}$ on a Bruker DRX 300 Spectrometer $\left({ }^{1} \mathrm{H}: 300.1 \mathrm{MHz},{ }^{13} \mathrm{C}: 75.4 \mathrm{MHz}\right)$ or on a Bruker Avance 600 Spectrometer $\left({ }^{1} \mathrm{H}: 600.6\right.$ $\mathrm{MHz},{ }^{13} \mathrm{C}: 151.0 \mathrm{MHz}$ ) at $295 \mathrm{~K}$. Chemical shifts $(\delta)$ are given in parts per million (ppm) downfield relative to TMS, and coupling constants $(J)$ are in $\mathrm{Hz}$. Residual solvent central signals were recorded as follows: $\mathrm{CDCl}_{3}, \delta_{\mathrm{H}}=$ 7.263, $\delta_{C}=77.00 ;$ DMSO- $d_{6}, \delta_{H}=2.50, \delta_{C}=39.43$. High-resolution mass spectra (HRMS) were recorded on a Waters LCD Premier XE instrument, and only the $[\mathrm{M}+\mathrm{H}]^{+}$or $[\mathrm{M}+\mathrm{Na}]^{+}$molecular species are reported. Selenium powder (100 mesh) with purity $\geq 99.5 \%$ used for $\mathrm{Li}_{2} \mathrm{Se}_{2}$ or $\mathrm{Na}_{2} \mathrm{Se}_{2}$ preparation was purchased from Aldrich. Freshly distilled acetonitrile (MeCN) was redistilled twice over $\mathrm{P}_{2} \mathrm{O}_{5}$ to obtain the anhydrous MeCN for preparation of the ebselen derivatives 4. Methylene chloride $\left(\mathrm{CH}_{2} \mathrm{Cl}_{2}\right)$ was distilled over $\mathrm{P}_{2} \mathrm{O}_{5}$ before preparation of diselenides 6 . Distilled triethylamine was stored over $\mathrm{NaOH}$ pellets. Other reagents and starting materials were directly used as obtained commercially. Purity of the products was confirmed by comparison of their melting point with data given in the literature and by spectroscopic methods.

2,2'-Diselenobisbenzoic acid (2) was prepared from anthranilic acid, using dilithium diselenide, ${ }^{30}$ following the method previously reported ${ }^{28}$ with some modifications. The crude product was recrystallized from 1,4-dioxane by slow evaporation under ca. $200 \mathrm{mmHg}$ from a water bath at ca $75{ }^{\circ} \mathrm{C}$ to give $2,2^{\prime}$-diselenobisbenzoic acid (2) with $85 \%$ yield as pale crystals, $\mathrm{mp} 299-300{ }^{\circ} \mathrm{C}$ (lit. ${ }^{30} 300-303{ }^{\circ} \mathrm{C}$ ).

2-(Chloroseleno)benzoyl chloride (3) was prepared from 2,2'-diselenobisbenzoic acid (2) using excess of thionyl chloride, following the method previously reported. ${ }^{29}$ Crude oily product was recrystallized from $n$ hexane and dried under vacuum $(200 \mathrm{mmHg})$ to give 2-(chloroseleno)benzoyl chloride (3) as yellow needles, $\mathrm{mp} 65-67^{\circ} \mathrm{C}$ (lit. $\left.{ }^{29} 65-66^{\circ} \mathrm{C}\right)$.

Bis[(2-chlorocarbonyl)phenyl)] diselenide (5) was prepared from 2,2'-diselenobisbenzoic acid (2), using thionyl chloride in excess of 2.5 equivalents, following the method previously reported. ${ }^{29}$ The crude oily product was washed with $n$-hexane, recrystallized from $\mathrm{CH}_{2} \mathrm{Cl}_{2}$ and dried under vacuum to give bis[(2chlorocarbonyl)phenyl)] diselenide (5) as pale greenish yellow prisms, mp $180-181^{\circ} \mathrm{C}$ (lit. $.^{29} 174-175{ }^{\circ} \mathrm{C}$ ). 
General synthetic procedure for benzisoselenazol-3(2H)-ones 4 . To a stirred solution of substituted aniline $(5.0 \mathrm{mmol})$ and dry triethylamine $(1.8 \mathrm{ml}, 1.02 \mathrm{~g}, 12.5 \mathrm{mmol})$ in anhydrous $\mathrm{MeCN}(50 \mathrm{~mL})$ a solution of (2chloroseleno)benzoyl chloride $3(1.27 \mathrm{~g}, 5.0 \mathrm{mmol}$ ) in anhydrous MeCN (25 mL) was slowly added dropwise for $1 \mathrm{~h}$. The reaction was continued for 1-48 h. The progress of reaction was monitored by TLC. After the reaction finished, the solvent was evaporated and water was added dropwise $(100 \mathrm{~mL})$ resulting in the product precipitation. Crude product was washed with water and $3.5 \% \mathrm{HCl}$ if TLC indicated unreacted amine, and left to dry on the air.

2-(4-Methoxy-2-methylphenyl)benzisoselenazol-3(2H)-one (4a). White flakes, yield $67 \%, \mathrm{mp} 179-180{ }^{\circ} \mathrm{C}$ (from acetonitrile-water, 1:1 v/v); ${ }^{1} \mathrm{H}$ NMR (300 MHz, DMSO- $\left.d_{6}\right): \delta_{\mathrm{H}} 2.07\left(\mathrm{~s}, 3 \mathrm{H}, \mathrm{CH}_{3}\right), 3.78\left(\mathrm{~s}, 3 \mathrm{H}, \mathrm{OCH}_{3}\right), 6.84$ (dd, J $\left.8.6 \mathrm{~Hz}, J 2.9 \mathrm{~Hz}, 1 \mathrm{H}, \mathrm{H}-5^{\prime}\right), 6.92\left(\mathrm{~d}, J 2.9 \mathrm{~Hz}, 1 \mathrm{H}, \mathrm{H}-3^{\prime}\right), 7.20\left(\mathrm{~d}, J 8.6 \mathrm{~Hz}, 1 \mathrm{H}, \mathrm{H}-6^{\prime}\right), 7.47$ (dd, J $8.3 \mathrm{~Hz}, J 6.9$ $\mathrm{Hz}, 1 \mathrm{H}, \mathrm{H}-6), 7.67$ (ddd, J $8.0 \mathrm{~Hz}, J 6.9 \mathrm{~Hz}, J 1.3 \mathrm{~Hz}, 1 \mathrm{H}, \mathrm{H}-5), 7.88$ (dd, J $8.3 \mathrm{~Hz}, J 1.3 \mathrm{~Hz}, 1 \mathrm{H}, \mathrm{H}-7$ ), 8.08 (d, J 8.0 $\mathrm{Hz}, 1 \mathrm{H}, \mathrm{H}-4) .{ }^{13} \mathrm{C}$ NMR $(75 \mathrm{MHz}$, DMSO-d $)$ : $\delta_{\mathrm{C}} 18.0,55.2,111.9,115.7,125.9,126.0,127.3,127.8,129.7,129.9$, 131.8, 137.9, 140.0, 158.8, 165.2. HRMS (TOF MS ESI): $m / z$ for $\left(\mathrm{C}_{15} \mathrm{H}_{13} \mathrm{NO}_{2} \mathrm{Se}+\mathrm{H}\right)^{+}$calculated: 320.0190 ; found: 320.0190.

2-(2-Methoxy-5-methylphenyl)benzisoselenazol-3(2H)-one (4b). Pale brown needles, yield 63\%, mp 172-173 ${ }^{\circ} \mathrm{C}$ (from water); ${ }^{1} \mathrm{H}$ NMR $\left(300 \mathrm{MHz}, \mathrm{DMSO}-d_{6}\right): \delta_{\mathrm{H}} 2.27\left(\mathrm{~s}, 3 \mathrm{H}, \mathrm{CH}_{3}\right), 3.73\left(\mathrm{~s}, 3 \mathrm{H}, \mathrm{OCH}_{3}\right), 7.03(\mathrm{~d}, 1 \mathrm{H}, J 6.4 \mathrm{~Hz}, \mathrm{H}-$ 3'), 7.16-7.18 (m, 2H, H-4', H-6'), 7.45 (ddd, J 7.9 Hz, J $7.5 \mathrm{~Hz}, J 0.8 \mathrm{~Hz}, 1 \mathrm{H}, \mathrm{H}-6), 7.66$ (ddd, J $8.2 \mathrm{~Hz}, J 7.0 \mathrm{~Hz}, J$ $1.4 \mathrm{~Hz}, 1 \mathrm{H}, \mathrm{H}-5), 7.86$ (dd, J $7.7 \mathrm{~Hz}, J 0.9 \mathrm{~Hz}, 1 \mathrm{H}, \mathrm{H}-7), 8.06$ (d, J $8.0 \mathrm{~Hz}, 1 \mathrm{H}, \mathrm{H}-4) .{ }^{13} \mathrm{C} \mathrm{NMR}\left(75 \mathrm{MHz}, \mathrm{DMSO}-d_{6}\right)$ : $\delta_{C} 19.8,55.7,112.5,125.7,125.8,126.8,127.3,127.7,129.3,129.4,130.0,131.8,140.3,153.0,165.7 . ~ H R M S$ (TOF MS ESI): $\mathrm{m} / \mathrm{z}$ for $\left(\mathrm{C}_{15} \mathrm{H}_{13} \mathrm{NO}_{2} \mathrm{Se}+\mathrm{H}\right)^{+}$calculated: 320.0190 ; found: 320.0201 .

2-(4-Chloro-3-methylphenyl)benzisoselenazol-3(2H)-one (4c). Shiny orange crystals, yield 70\%, mp 209-210 ${ }^{\circ} \mathrm{C}$ (from water); ${ }^{1} \mathrm{H}$ NMR (300 MHz, DMSO- $\left.d_{6}\right): \delta_{\mathrm{H}} 2.38\left(\mathrm{~s}, 3 \mathrm{H}, \mathrm{CH}_{3}\right), 7.47-7.53\left(\mathrm{~m}, 3 \mathrm{H}, \mathrm{H}-2^{\prime}, 5^{\prime}, 6^{\prime}\right), 7.66-7.73(\mathrm{~m}$, $2 \mathrm{H}, \mathrm{H}-5,6), 7.91$ (d, J $7.7 \mathrm{~Hz}, 1 \mathrm{H}, \mathrm{H}-7), 8.10$ (d, J 8.0 Hz, 1H, H-4). ${ }^{13} \mathrm{C}$ NMR (75 MHz, DMSO-d $\left.)^{2}\right): \delta_{c} 19.6,123.6$, $125.8,126.2,127.0,127.9,128.3,129.3,130.0,132.3,136.3,138.5,138.8,165.0$. HRMS (TOF MS ESI): $\mathrm{m} / z$ for $\left(\mathrm{C}_{14} \mathrm{H}_{10} \mathrm{ClNOSe}+\mathrm{H}\right)^{+}$calculated: 323.9692 ; found: 323.9692 .

2-(3-Chloro-2-methylphenyl)benzisoselenazol-3(2H)-one (4d). Yellow crystals, yield 55\%, mp $282-283{ }^{\circ} \mathrm{C}$

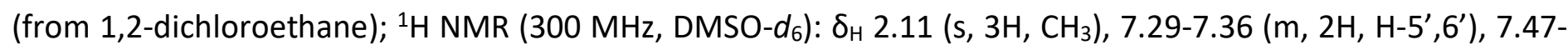
$7.54\left(\mathrm{~m}, 2 \mathrm{H}, \mathrm{H}-6,4^{\prime}\right.$ ), 7.70 (ddd, J $8.0 \mathrm{~Hz}, J 7.0 \mathrm{~Hz}, J 1.4 \mathrm{~Hz}, 1 \mathrm{H}, \mathrm{H}-5$ ), 7.90 (dd, J 7.7 Hz, J $1.2 \mathrm{~Hz}, 1 \mathrm{H}, \mathrm{H}-7$ ), 8.10 (d, J $8.0 \mathrm{~Hz}, 1 \mathrm{H}, \mathrm{H}-4) .{ }^{13} \mathrm{C}$ NMR $\left(75 \mathrm{MHz}, \mathrm{DMSO}-d_{6}\right): \delta_{c} 15.3,126.0,126.1,126.9,127.5,127.9,128.0,128.7,132.1$, 134.1, 134.8, 139.0, 140.2, 165.3. HRMS (TOF MS ESI): $m / z$ for $\left(\mathrm{C}_{14} \mathrm{H}_{10} \mathrm{CINOSe}+\mathrm{H}\right)^{+}$calculated: 323.9692; found: 323.9685 .

2-(5-Chloro-2-methoxyphenyl)benzisoselenazol-3(2H)-one (4e). Light yellow prisms, yield 79\%, mp 205-206 ${ }^{\circ} \mathrm{C}$ (from acetonitrile-water, 85:15 v/v); ${ }^{1} \mathrm{H}$ NMR $\left(300 \mathrm{MHz}, \mathrm{DMSO}-d_{6}\right): \delta_{\mathrm{H}} 3.79\left(\mathrm{~s}, 3 \mathrm{H}, \mathrm{OCH}_{3}\right), 7.19(\mathrm{~d}, J 8.9 \mathrm{~Hz}$, $1 \mathrm{H}, \mathrm{H}-3^{\prime}$ ), 7.41-7.49 (m, 3H, H-6,4',6'), 7.67 (ddd, J $8.2 \mathrm{~Hz}, J 7.2 \mathrm{~Hz}, J 1.4 \mathrm{~Hz}, 1 \mathrm{H}, \mathrm{H}-5$ ), 7.87 (dd, J $7.8 \mathrm{~Hz}, J 0.8 \mathrm{~Hz}$, $1 \mathrm{H}, \mathrm{H}-7), 8.07$ (d, J $8.1 \mathrm{~Hz}, 1 \mathrm{H}, \mathrm{H}-4) .{ }^{13} \mathrm{C} \mathrm{NMR}\left(75 \mathrm{MHz}, \mathrm{DMSO}-d_{6}\right): \delta_{\mathrm{C}} 56.6,114.6,124.1,126.3,126.4,127.5$, 128.4, 129.0, 129.1, 129.6, 132.6, 141.0, 154.6, 166.5. HRMS (TOF MS ESI): $\mathrm{m} / \mathrm{z}$ for $\left(\mathrm{C}_{14} \mathrm{H}_{10} \mathrm{CINO}_{2} \mathrm{Se}+\mathrm{H}\right)^{+}$ calculated: 339.9641; found: 339.9636.

2-(5-t-Butyl-2-methoxyphenyl)benzisoselenazol-3(2H)-one (4f). Purple powder, yield 82\%, mp 207-208 ${ }^{\circ} \mathrm{C}$ (purified by column chromatography, chloroform); ${ }^{1} \mathrm{H} N M R(300 \mathrm{MHz}, \mathrm{CDCl} 3): \delta_{\mathrm{H}} 1.31$ (s, $\left.9 \mathrm{H}, t-\mathrm{Bu}\right), 3.82(\mathrm{~s}, 3 \mathrm{H}$, $\left.\mathrm{OCH}_{3}\right), 6.94\left(\mathrm{~d}, J 8.7 \mathrm{~Hz}, 1 \mathrm{H}, \mathrm{H}-3^{\prime}\right), 7.37$ (dd, J 8.7 Hz, J $\left.2.5 \mathrm{~Hz}, 1 \mathrm{H}, \mathrm{H}-4^{\prime}\right), 7.42-7.47$ (m, 2H, H-6,6'), 7.62-7.65 (m, $2 \mathrm{H}, \mathrm{H}-5,7), 8.12$ (d, J $7.6 \mathrm{~Hz}, 1 \mathrm{H}, \mathrm{H}-4) .{ }^{13} \mathrm{C} N M R\left(75 \mathrm{MHz}, \mathrm{DMSO}-d_{6}\right): \delta_{c} 31.7,33.7,55.7,112.2,125.6(2 \mathrm{C})$, 125.7, 126.2, 126.5, 127.3, 127.7, 131.8, 140.3, 142.9, 152.9, 165.7. HRMS (TOF MS ESI): $m / z$ for $\left(\mathrm{C}_{18} \mathrm{H}_{19} \mathrm{NO}_{2} \mathrm{Se}\right.$ $+\mathrm{H})^{+}$calculated: 362.0660; found: 362.0659 . 
2-(2-Chloro-5-methylphenyl)benzisoselenazol-3(2H)-one $\mathbf{( 4 g )}$. White powder, yield 70\%, mp $184-185{ }^{\circ} \mathrm{C}$ (purified by column chromatography (chloroform/ethyl acetate, $5: 1) ;{ }^{1} \mathrm{H} \mathrm{NMR}\left(300 \mathrm{MHz}, \mathrm{CDCl}_{3}\right): \delta_{\mathrm{H}} 2.35(\mathrm{~s}, 3 \mathrm{H}$, $\left.\mathrm{CH}_{3}\right), 7.16\left(\mathrm{~d}, J 8.2 \mathrm{~Hz}, 1 \mathrm{H}, \mathrm{H}-4^{\prime}\right), 7.27(\mathrm{~s}, 1 \mathrm{H}, \mathrm{H}-6), 7.38$ (d, J $\left.8.2 \mathrm{~Hz}, 1 \mathrm{H}, \mathrm{H}-3^{\prime}\right), 7.47$ (dd, J 7.6 Hz, J $6.8 \mathrm{~Hz}, 1 \mathrm{H}, \mathrm{H}-$ 5), 7.62-7.70 (m, 2H, H-6,7), 8.13 (d, J 7.6 Hz, 1H, H-4). ${ }^{13} \mathrm{C} \mathrm{NMR} \mathrm{(75} \mathrm{MHz,} \mathrm{CDCl} 3$ ): $\delta_{\mathrm{c}} 20.5,124.4,125.8,126.1$, $129.0,129.9,130.4,130.6,131.4,132.3,135.1,137.8,139.5,166.6$. HRMS (TOF MS ESI): $\mathrm{m} / z$ for $\mathrm{C}_{14} \mathrm{H}_{10} \mathrm{ClNOSe}+\mathrm{H}^{+}$calculated: 323.9692; found: 323.9607.

2-(2-Chloro-4-methylphenyl)benzisoselenazol-3(2H)-one (4h). White powder, yield 95\%, $\mathrm{mp} 208-209{ }^{\circ} \mathrm{C}$ (from ethyl acetate); ${ }^{1} \mathrm{H} N M R\left(300 \mathrm{MHz}, \mathrm{CDCl}_{3}\right): \delta_{\mathrm{H}} 2.36\left(\mathrm{~s}, 3 \mathrm{H}, \mathrm{CH}_{3}\right), 7.25\left(\mathrm{dd}, J 8.0 \mathrm{~Hz}, J 1.1 \mathrm{~Hz}, 1 \mathrm{H}, \mathrm{H}-5^{\prime}\right), 7.37$ (d, J $\left.8.0 \mathrm{~Hz}, 1 \mathrm{H}, \mathrm{H}-6^{\prime}\right), 7.45-7.50\left(\mathrm{~m}, 2 \mathrm{H}, \mathrm{H}-6,3^{\prime}\right), 7.69$ (ddd, J $\left.8.0 \mathrm{~Hz}, J 7.3 \mathrm{~Hz}, J 1.3 \mathrm{~Hz}, 1 \mathrm{H}, \mathrm{H}-5\right), 7.89$ (dd, J 7.7 $\mathrm{Hz}, J 0.8 \mathrm{~Hz}, 1 \mathrm{H}, \mathrm{H}-7), 8.09$ (d, J $8.0 \mathrm{~Hz}, 1 \mathrm{H}, \mathrm{H}-4) .{ }^{13} \mathrm{C} N M R\left(75 \mathrm{MHz}, \mathrm{DMSO}-d_{6}\right): \delta_{\mathrm{C}} 20.3,125.9,126.0,126.8$, $127.9,128.5,130.3,130.6,132.1$ (2C), 133.3, 139.8, 140.3, 165.6. HRMS (TOF MS ESI): $\mathrm{m} / \mathrm{z}$ for $\mathrm{C}_{14} \mathrm{H}_{10} \mathrm{ClNOSe}+\mathrm{H}^{+}$calculated: 323.9692; found: 323.9694 .

2-(4-Methylphenyl)benzisoselenazol-3(2H)-one (4i). Pale powder, yield $72 \%, \mathrm{mp} 163-164{ }^{\circ} \mathrm{C}$ (from water) (lit. $\left.{ }^{33} 163-164{ }^{\circ} \mathrm{C}\right) ;{ }^{1} \mathrm{H}$ NMR $\left(300 \mathrm{MHz}, \mathrm{DMSO}-d_{6}\right): \delta_{\mathrm{H}} 2.32\left(\mathrm{~s}, 3 \mathrm{H}, \mathrm{CH}_{3}\right), 7.24$ (d, J $\left.8.1 \mathrm{~Hz}, 2 \mathrm{H}, \mathrm{H}-2^{\prime}, 6^{\prime}\right), 7.47-7.53$ (m, 3H, H-6,3', 5'), 7.67 (ddd, J $8.0 \mathrm{~Hz}, J 7.5 \mathrm{~Hz}, J 1.3 \mathrm{~Hz}, 1 \mathrm{H}, \mathrm{H}-5), 7.90$ (d, J 7.0 Hz, 1H, H-7), 8.09 (d, J $8.0 \mathrm{~Hz}$, $1 \mathrm{H}, \mathrm{H}-4) .{ }^{13} \mathrm{C}$ NMR $\left(75 \mathrm{MHz}\right.$, DMSO- $\left.d_{6}\right): \delta_{c} 20.5,124.5,125.7,126.2,127.8,128.4,129.5,132.0,135.1,137.0$, 138.8, 164.8. HRMS (TOF MS ESI): $\mathrm{m} / z$ for $\left(\mathrm{C}_{14} \mathrm{H}_{11} \mathrm{NOSe}+\mathrm{H}\right)^{+}$calculated: 290.0085 ; found: 290.0084 .

2-(5-chloro-2-methylphenyl)benzisoselenazol-3(2H)-one (4j). White powder, yield 60\%, mp $189-190{ }^{\circ} \mathrm{C}$ (from chloroform); ${ }^{1} \mathrm{H}$ NMR (300 MHz, DMSO- $\left.d_{6}\right): \delta_{\mathrm{H}} 2.08\left(\mathrm{~s}, 3 \mathrm{H}, \mathrm{CH}_{3}\right), 7.36-7.42\left(\mathrm{~m}, 3 \mathrm{H}, \mathrm{H}-3^{\prime}, 4^{\prime}, 6^{\prime}\right), 7.49(\mathrm{dd}, J 7.5 \mathrm{~Hz}$, J $7.3 \mathrm{~Hz}, 1 \mathrm{H}, \mathrm{H}-6$ ), 7.69 (ddd, J $8.0 \mathrm{~Hz}, J 7.3 \mathrm{~Hz}, J 1.3 \mathrm{~Hz}, 1 \mathrm{H}, \mathrm{H}-5), 7.89$ (d, J $7.6 \mathrm{~Hz}, 1 \mathrm{H}, \mathrm{H}-7$ ), 8.10 (d, J $8.0 \mathrm{~Hz}, 1 \mathrm{H}$, $\mathrm{H}-4) .{ }^{13} \mathrm{C}$ NMR $\left(151 \mathrm{MHz}, \mathrm{DMSO}-d_{6}\right): \delta_{\mathrm{C}} 17.8,126.5,126.6,127.5,128.4,128.5,128.6,129.1,130.8,132.6$, 132.7, 139.4, 140.8, 165.8. HRMS (TOF MS ESI): $\mathrm{m} / \mathrm{z}$ for $\left(\mathrm{C}_{14} \mathrm{H}_{10} \mathrm{CINOSe}+\mathrm{H}\right)^{+}$calculated: $323.9692 ;$ found: 323.9687.

2-(3-Methoxyphenyl)benzisoselenazol-3(2H)-one (4k). Pale yellow crystals, yield $63 \%, \mathrm{mp} 165.5-167.5{ }^{\circ} \mathrm{C}$ (from acetonitrile) (lit. ${ }^{34} 162-164{ }^{\circ} \mathrm{C}$ ); ${ }^{1} \mathrm{H}$ NMR (300 MHz, DMSO- $\left.d_{6}\right): \delta_{\mathrm{H}} 3.77\left(\mathrm{~s}, 3 \mathrm{H}, \mathrm{OCH}_{3}\right), 6.82(\mathrm{dd}, J 8.3 \mathrm{~Hz}, J$ $\left.0.8 \mathrm{~Hz}, 1 \mathrm{H}, \mathrm{H}-6^{\prime}\right), 7.15\left(\mathrm{dd}, J 8.0 \mathrm{~Hz}, J 0.8 \mathrm{~Hz}, 1 \mathrm{H}, \mathrm{H}-4^{\prime}\right), 7.31-7.36\left(\mathrm{~m}, 2 \mathrm{H}, \mathrm{H}-2^{\prime}, 5^{\prime}\right), 7.45$ (ddd, J $7.8 \mathrm{~Hz}, J 7.2 \mathrm{~Hz}, J$ $0.9 \mathrm{~Hz}, 1 \mathrm{H}, \mathrm{H}-6), 7.64$ (ddd, J $8.0 \mathrm{~Hz}, J 7.2 \mathrm{~Hz}, J 1.4 \mathrm{~Hz}, 1 \mathrm{H}, \mathrm{H}-5), 7.88$ (dd, J $7.7 \mathrm{~Hz}, J 0.9 \mathrm{~Hz}, 1 \mathrm{H}, \mathrm{H}-7$ ), 8.36 (d, J $8.0 \mathrm{~Hz}, 1 \mathrm{H}, \mathrm{H}-4) .{ }^{13} \mathrm{C}$ NMR $\left(75 \mathrm{MHz}\right.$, DMSO- $\left.d_{6}\right): \delta_{\mathrm{C}} 55.2,110.3,111.3,116.6,125.7,126.2,127.9,128.5,129.9$, 132.2, 138.8, 140.8, 159.6, 164.9. HRMS (TOF MS ESI): $\mathrm{m} / \mathrm{z}$ for $\left(\mathrm{C}_{14} \mathrm{H}_{11} \mathrm{NO}_{2} \mathrm{Se}+\mathrm{H}\right)^{+}$calculated: 306.0034; found: 306.0030 .

2-(4-Methoxyphenyl)benzisoselenazol-3(2H)-one (4I). Pale needles, yield $70 \%, \mathrm{mp} 180.5-181.5{ }^{\circ} \mathrm{C}$ (from water) (lit. $\left.{ }^{28} 181-184{ }^{\circ} \mathrm{C}\right) ;{ }^{1} \mathrm{H}$ NMR $\left(300 \mathrm{MHz}, \mathrm{DMSO}-d_{6}\right): \delta_{\mathrm{H}} 3.78\left(\mathrm{~s}, 3 \mathrm{H}, \mathrm{OCH}_{3}\right), 7.01(\mathrm{dd}, J 9.0 \mathrm{~Hz}, J 2.3 \mathrm{~Hz}, 2 \mathrm{H}$, H-3', ,' $), 7.47$ (ddd, J $7.8 \mathrm{~Hz}, J 7.1 \mathrm{~Hz}, J 1.0 \mathrm{~Hz}, 1 \mathrm{H}, \mathrm{H}-6), 7.50$ (dd, J $9.0 \mathrm{~Hz}, J 2.3 \mathrm{~Hz}, 2 \mathrm{H}, \mathrm{H}-2^{\prime}, 6^{\prime}$ ), 7.67 (ddd, J 7.9 $\mathrm{Hz}, J 7.2 \mathrm{~Hz}, J 1.4 \mathrm{~Hz}, 1 \mathrm{H}, \mathrm{H}-5), 7.89$ (dd, J $7.8 \mathrm{~Hz}, J 0.8 \mathrm{~Hz}, 1 \mathrm{H}, \mathrm{H}-7), 8.08$ (d, J $7.9 \mathrm{~Hz}, 1 \mathrm{H}, \mathrm{H}-4) .{ }^{13} \mathrm{C}$ NMR (75 $\left.\mathrm{MHz}, \mathrm{DMSO}-d_{6}\right): \delta_{\mathrm{C}} 55.3,114.3(2 \mathrm{C}), 125.7,126.1,126.5(2 \mathrm{C}), 127.8,128.2,131.9,132.1,138.9,157.2,164.9$. HRMS (TOF MS ESI): $\mathrm{m} / \mathrm{z}$ for $\left(\mathrm{C}_{14} \mathrm{H}_{11} \mathrm{NO}_{2} \mathrm{Se}+\mathrm{H}\right)^{+}$calculated: 306.0034 ; found: 306.0039 .

General synthetic procedure for bis(2-carbamoylaryl) diselenides 6. To a stirred solution of substituted aniline $(1.0 \mathrm{mmol})$ in dry $\mathrm{CH}_{2} \mathrm{Cl}_{2}(5 \mathrm{~mL}) \mathrm{Na}_{2} \mathrm{CO}_{3}(2.5 \mathrm{mmol}, 0.265 \mathrm{~g})$ was added. Next, the solution of chloride 5 in dry $\mathrm{CH}_{2} \mathrm{Cl}_{2}$ was added dropwise for $30 \mathrm{~min}$. The desired product, diselenide precipitated immediately. The reaction progress was controlled by TLC. The stirring was continued for 1-48 $\mathrm{h}$. The product was filtered off and washed with dry $\mathrm{CH}_{2} \mathrm{Cl}_{2}(2 \times 5 \mathrm{~mL})$, water $(1 \times 5 \mathrm{~mL})$ and $3.5 \% \mathrm{HCl}(3 \times 5 \mathrm{~mL})$.

Bis[2-(4-chloro-3-methylphenylcarbamoyl)]phenyl diselenide (6c). White solid, yield 95\%, $\mathrm{mp} 239-240{ }^{\circ} \mathrm{C}$ (from 1,2-dichloroethane); ${ }^{1} \mathrm{H}$ NMR (300 MHz, DMSO-d $)_{6}$ : $\delta_{\mathrm{H}} 2.35\left(\mathrm{~s}, 6 \mathrm{H}, \mathrm{CH}_{3}\right), 7.38-7.50(\mathrm{~m}, 6 \mathrm{H}, \mathrm{ArH}-5, \mathrm{PhH}-$ 
4,5), 7.63 (d, J $8.6 \mathrm{~Hz}, 2 \mathrm{H}, \mathrm{ArH}-6), 7.76-7.82$ (m, 4H, PhH-6, ArH-2), 7.96 (d, J 7.2 Hz, 2H, PhH-3), 10.61 (s, 2H, $\mathrm{NH}) .{ }^{13} \mathrm{C}$ NMR (151 MHz, DMSO- $\left.d_{6}\right): \delta_{c} 19.81\left(\mathrm{CH}_{3}\right), 119.64(\mathrm{CH}), 122.83(\mathrm{CH}), 126.37(\mathrm{CH}), 128.01(\mathrm{C}), 128.62$ (CH), $128.93(\mathrm{CH}), 130.14(\mathrm{CH}), 131.99$ (C), $132.07(\mathrm{CH}), 133.45(\mathrm{C}), 135.54(\mathrm{C}), 137.48(\mathrm{C}), 166.26$ (C=O). HRMS (TOF MS ESI): $\mathrm{m} / z$ for $\left(\mathrm{C}_{28} \mathrm{H}_{21} \mathrm{Cl}_{2} \mathrm{~N}_{2} \mathrm{O}_{2} \mathrm{Se}_{2}+\mathrm{Na}\right)^{+}$calculated: 668.9297, 670.9284, 672.9272; found: 668.9341, $670.9288,672.9265$.

Bis[2-(5-chloro-2-methoxyphenylcarbamoyl)]phenyl diselenide (6e). Grey powder, yield 85\%, mp $194-195{ }^{\circ} \mathrm{C}$ (from methylene chloride); ${ }^{1} \mathrm{H}$ NMR $\left(300 \mathrm{MHz}, \mathrm{DMSO}-d_{6}\right): \delta_{\mathrm{H}} 3.86\left(\mathrm{~s}, 6 \mathrm{H}, \mathrm{OCH}_{3}\right), 7.15(\mathrm{~d}, J 8.9 \mathrm{~Hz}, 2 \mathrm{H}, \mathrm{ArH}-3)$, 7.29 (dd, J $2.6 \mathrm{~Hz}, J 8.8 \mathrm{~Hz}, 2 \mathrm{H}, \mathrm{ArH}-4), 7.38-7.51$ (m, 4H, PhH-4,5), 7.78 (d, J 7.8 Hz, 2H, PhH-6), 7.84 (d, J 2.6 $\mathrm{Hz}, 2 \mathrm{H}, \mathrm{ArH}-6), 7.99$ (d, J $7.5 \mathrm{~Hz}, 2 \mathrm{H}, \mathrm{PhH}-3$ ), 9.93 (s, 2H, NH). HRMS (TOF MS ESI): $m / z$ for $\left(\mathrm{C}_{28} \mathrm{H}_{22} \mathrm{Cl}_{2} \mathrm{~N}_{2} \mathrm{O}_{4} \mathrm{Se}_{2}+\right.$ $\mathrm{H})^{+}$calculated: 680.9363 ; found: 680.9400 .

Bis[2-(2-chloro-5-methylphenylcarbamoyl)]phenyl diselenide (6g). White solid, yield 75\%, $\mathrm{mp} 203-204{ }^{\circ} \mathrm{C}$ (from methylene chloride); ${ }^{1} \mathrm{H}$ NMR $\left(300 \mathrm{MHz}, \mathrm{DMSO}-d_{6}\right): \delta_{\mathrm{H}} 2.35\left(\mathrm{~s}, 6 \mathrm{H}, \mathrm{CH}_{3}\right), 7.18(\mathrm{dd}, J 8.1 \mathrm{~Hz}, J 1.1 \mathrm{~Hz}, 2 \mathrm{H}$, ArH-4), 7.43-7.46 (m, 4H, PhH-5, ArH-6), 7.47 (d, J 8.2 Hz, 2H, ArH-3), 7.50 (dd, J 7.4 Hz, J 1.0 Hz, 2H, PhH-4), $7.80(\mathrm{~d}, J 8.0 \mathrm{~Hz}, 2 \mathrm{H}, \mathrm{PhH}-6), 8.06$ (d, J $7.5 \mathrm{~Hz}, 2 \mathrm{H}, \mathrm{PhH}-3), 10.36(\mathrm{~s}, 2 \mathrm{H}, \mathrm{NH}) .{ }^{13} \mathrm{C} N M R\left(75 \mathrm{MHz}, \mathrm{DMSO}-d_{6}\right): \delta_{c}$ 20.4, 126.5, 126.7, 128.6, 128.7, 129.0, 129.3, 130.2, 132.3, 132.5, 132.6, 134.2, 137.3, 166.5. HRMS (TOF MS ESI): $m / z$ for $\left(\mathrm{C}_{28} \mathrm{H}_{21} \mathrm{Cl}_{2} \mathrm{~N}_{2} \mathrm{O}_{2} \mathrm{Se}_{2}+\mathrm{Na}\right)^{+}$calculated: 670.9284 ; found: 670.9287 .

Bis[2-(2-chloro-4-methylphenylcarbamoyl)]phenyl diselenide (6h). White powder, yield $62 \%, \mathrm{mp} 260-261{ }^{\circ} \mathrm{C}$ (from methylene chloride); ${ }^{1} \mathrm{H}$ NMR (300 MHz, DMSO- $\left.d_{6}\right): \delta_{\mathrm{H}} 2.35\left(\mathrm{~s}, 6 \mathrm{H}, \mathrm{CH}_{3}\right), 7.23(\mathrm{~d}, J 8.4 \mathrm{~Hz}, 2 \mathrm{H}, \mathrm{ArH}-5)$, 7.40-7.51 (m, 8H, PhH-4,5, ArH-3,6), 7.77 (d, J 7.4 Hz, 2H, PhH-6), 8.05 (d, J 6.9 Hz, 2H, PhH-3), 10.35 (s, 2H, $\mathrm{NH}) .{ }^{13} \mathrm{C}$ NMR $\left(151 \mathrm{MHz}\right.$, DMSO- $\left.d_{6}\right): \delta_{c} 20.8,126.9,128.7,129.0,129.1,130.1,130.3,130.6,132.3,132.7$, 133.0, 133.1, 138.3, 167.0. HRMS (TOF MS ESI): $m / z$ for $\left(\mathrm{C}_{28} \mathrm{H}_{22} \mathrm{Cl}_{2} \mathrm{~N}_{2} \mathrm{O}_{2} \mathrm{Se}_{2}+\mathrm{H}\right)^{+}$calculated: 648.9465; found: 648.9327.

Bis[2-(5-chloro-2-methylphenylcarbamoyl)]phenyl diselenide (6j). White powder, yield $86 \%, \mathrm{mp} 247-248{ }^{\circ} \mathrm{C}$ (from 1,2-dichloroethane); ${ }^{1} \mathrm{H}$ NMR $\left(300 \mathrm{MHz}, \mathrm{DMSO}-d_{6}\right): \delta_{\mathrm{H}} 2.28\left(\mathrm{~s}, 6 \mathrm{H}, \mathrm{CH}_{3}\right), 7.28$ (dd, J 8.3 Hz, J $2.2 \mathrm{~Hz}, 2 \mathrm{H}$, ArH-4), 7.34 (d, J $8.3 \mathrm{~Hz}, 2 \mathrm{H}, \mathrm{ArH}-3), 7.40-7.52$ (m, 4H, PhH-4,5), 7.53 (d, J 2.2 Hz, 2H, ArH-6), 7.80 (dd, J 7.8 Hz, J $1.3 \mathrm{~Hz}, 2 \mathrm{H}, \mathrm{PhH}-6$ ), 8.02 (dd, J $7.4 \mathrm{~Hz}, J 1.4 \mathrm{~Hz}, 2 \mathrm{H}, \mathrm{PhH}-3$ ), 10.28 (s, 2H, NH). ${ }^{13} \mathrm{C}$ NMR (75 MHz, DMSO- $\left.d_{6}\right): \delta_{c}$ $17.4,125.85,125.9,126.4,128.6,129.8,130.1,131.9,132.1,132.3,132.5,132.9,137.2,166.5$. HRMS (TOF MS ESI): $m / z$ for $\left(\mathrm{C}_{28} \mathrm{H}_{21} \mathrm{Cl}_{2} \mathrm{~N}_{2} \mathrm{O}_{2} \mathrm{Se}_{2}+\mathrm{Na}\right)^{+}$calculated: 670.9284 ; found: 670.9291 .

Bis[2-(3-methoxyphenylcarbamoyl)]phenyl diselenide (6k). White powder, yield 74\%, mp $221-223{ }^{\circ} \mathrm{C}$ (from methylene chloride); ${ }^{1} \mathrm{H} \mathrm{NMR}\left(300 \mathrm{MHz}, \mathrm{DMSO}-d_{6}\right): \delta_{\mathrm{H}} 3.78\left(\mathrm{~s}, 6 \mathrm{H}, \mathrm{OCH}_{3}\right), 6.74(\mathrm{dd}, J 8.2 \mathrm{~Hz}, J 1.7 \mathrm{~Hz}, 2 \mathrm{H}, \mathrm{ArH}-$ 4), 7.30 (dd, J $8.2 \mathrm{~Hz}, J 8.1 \mathrm{~Hz}, 2 \mathrm{H}, \mathrm{ArH}-5), 7.40$ (dd, J $8.1 \mathrm{~Hz}, J 1.2 \mathrm{~Hz}, 2 \mathrm{H}, \mathrm{ArH}-6), 7.42$ (ddd, J $8.0 \mathrm{~Hz}, J 7.0 \mathrm{~Hz}, J$ $1.2 \mathrm{~Hz}, 2 \mathrm{H}, \mathrm{PhH}-5), 7.45-7.48$ (m, 4H, PhH-4, ArH-2), 7.81 (dd, J $8.0 \mathrm{~Hz}, J 1.1 \mathrm{~Hz}, 2 \mathrm{H}, \mathrm{PhH}-6), 7.96$ (dd, J $7.6 \mathrm{~Hz}, J$ $1.3 \mathrm{~Hz}, 2 \mathrm{H}, \mathrm{PhH}-3), 10.54(\mathrm{~s}, 2 \mathrm{H}, \mathrm{NH}) .{ }^{13} \mathrm{C}$ NMR $\left(151 \mathrm{MHz}, \mathrm{DMSO}-d_{6}\right): \delta_{\mathrm{C}} 55.0,106.2,109.5,112.7,126.4,128.6$, 129.5, 130.1, 131.9, 132.0, 133.7, 139.8, 159.4, 166.3. HRMS (TOF MS ESI): $m / z$ for $\left(\mathrm{C}_{28} \mathrm{H}_{24} \mathrm{~N}_{2} \mathrm{O}_{4} \mathrm{Se}_{2}+\mathrm{H}\right)^{+}$ calculated: 612.0189; found: 612.0232 .

Bis[2-(4-methoxyphenylcarbamoyl)]phenyl diselenide (6I). White powder, yield 62\%, mp $285.5-287.5{ }^{\circ} \mathrm{C}$ (from methylene chloride) (lit. ${ }^{35} 290-292{ }^{\circ} \mathrm{C} ;{ }^{1} \mathrm{H}$ NMR $\left(300 \mathrm{MHz}, \mathrm{DMSO}-d_{6}\right): \delta_{\mathrm{H}} 3.76\left(\mathrm{~s}, 6 \mathrm{H}, \mathrm{OCH}_{3}\right), 6.97$ (d, J 9.0 $\mathrm{Hz}, 4 \mathrm{H}, \mathrm{ArH}-3,5$ ), 7.40 (ddd, J $7.1 \mathrm{~Hz}, J 6.9 \mathrm{~Hz}, J 0.9 \mathrm{~Hz}, 2 \mathrm{H}, \mathrm{PhH}-4$ ), 7.44 (ddd, J $8.0 \mathrm{~Hz}, J 7.1 \mathrm{~Hz}, J 1.3 \mathrm{~Hz}, 2 \mathrm{H}$, PhH-5), 7.68 (d, J $9.0 \mathrm{~Hz}, 4 \mathrm{H}, \mathrm{ArH}-2,6), 7.79$ (dd, J $8.0 \mathrm{~Hz}, J 0.8 \mathrm{~Hz}, 2 \mathrm{H}, \mathrm{PhH}-6), 7.95$ (d, J 6.8 Hz, 2H, PhH-3), $10.44(\mathrm{~s}, 2 \mathrm{H}, \mathrm{NH}) .{ }^{13} \mathrm{C}$ NMR $\left(151 \mathrm{MHz}, \mathrm{DMSO}-d_{6}\right): \delta_{c} 55.7,114.3,122.7,126.9,129.0,130.6,132.2,132.4$, 132.5, 134.3, 156.4, 166.4. HRMS (TOF MS ESI): $\mathrm{m} / z$ for $\left(\mathrm{C}_{28} \mathrm{H}_{24} \mathrm{~N}_{2} \mathrm{O}_{4} \mathrm{Se}_{2}+\mathrm{H}\right)^{+}$calculated: 612.0189; found: 612.01823. 
Evaluation of antiviral activity and cytotoxicity. The compounds $\mathbf{4}$ and $\mathbf{6}$ at various concentrations were incubated with following viruses: HHV-1 (human herpes virus type 1), EMCV (encephalomyocarditis virus) and VSV (vesicular stomatitis virus) for $1 \mathrm{hr}$ at RT. The HHV-1 was used at the dose of $10^{6} \mathrm{TCID}_{50} / \mathrm{mL}$, whereas EMCV and VSV at the dose of $10^{8} \mathrm{TCID}_{50} / \mathrm{mL}$. The $\mathrm{TCID}_{50}$ (tissue culture infectious dose) determines the cytopathic effect caused by the virus in about $50 \%$ of infected cells. The virus titer was measured in human cell line A549 (human lung adenocarcinoma cell line, ATCC 185) which was observed for cytopathic effect after 48 h. The minimal concentration that caused 1000 -fold decrease of virus titer was taken as minimal inhibitory concentration, MIC $(\mu \mathrm{g} / \mathrm{mL})$.

In parallel, the cytotoxicity of the compounds was determined in A549 cell line (cell line used in antiviral activity test) by incubation at various concentrations for $48 \mathrm{~h}$ at $37{ }^{\circ} \mathrm{C}$ in the atmosphere of $5 \% \mathrm{CO}_{2}$ in air. The minimal concentration which was toxic to approximately $50 \%$ of the cells was taken as $\mathrm{TCCD}_{50}$.

Both, cytotoxicity and virus titers were determined using MTT assay. To ensure that colourful compounds did not interfere with MTT reagents, extent of cell damage was evaluated by microscopic examination prior to MTT assay.

Evaluation of antimicrobial activity. Antimicrobial activity of compounds $\mathbf{4}$ and $\mathbf{6}$ was determined by serial dilution method using gram-positive bacterial strains: Staphylococcus aureus PCM 1944, Bacillus subtilis PCM 1949, gram-negative bacterial strains: Escherichia coli PCM 2057, Pseudomonas aeruginosa PCM 2058 and yeast Candida albicans ATCC 90028 obtained from the Polish Collection of Microorganisms, following procedure previously described. ${ }^{26}$ The antimicrobial activity was characterized by MIC value which is minimal concentration of the compound in $\mu \mathrm{g} / \mathrm{ml}$ that totally inhibits growth of the microorganisms.

\section{Acknowledgements}

This work was supported by the National Science Centre (NCN) through grant no. UMO-2013/09/D/ST5/03814 (antimicrobial studies of organoselenium compounds) and a statutory activity of subsidy from the Polish Ministry of Science and Higher Education for the Faculty of Chemistry of Wrocław University of Science and Technology (synthesis of organoselenium compounds).

\section{References}

1. Młochowski, J.; Wójtowicz-Młochowska, H. Molecules 2015, 20, 10205-10243 and references therein. http://dx.doi.org/10.3390/molecules200610205

2. Młochowski, J.; Kloc. K.; Lisiak, R.; Potaczek, P.; Wójtowicz, H. Arkivoc 2007, (vi), 14-46 and references therein. http://dx.doi.org/10.3998/ark.5550190.0008.603

3. Wirth, T. Angew. Chem. Int. Ed. 2000, 39, 3740-3749 and references therein. http://dx.doi.org/10.1002/1521-3773(20001103)39:21<3740::AID-ANIE3740>3.0.CO;2-N

4. Freudendahl, D. M; Santoro, S.; Shahzad, S. A.; Santi, C.; Wirth, T. Angew. Chem. Int. Ed. 2009, 48, 84098411 and references therein.

http://dx.doi.org/10.1002/anie.200903893 
5. Guo, R.; Huang, J.; Huang, H.; Zhao, X. Org. Lett. 2016, $18,504-507$. http://dx.doi.org/10.1021/acs.orglett.5b03543

6. Kumar, A.; Rao, G. K.; Saleem, F.; Singh, A. K. Dalton Trans. 2012, 41, 11949-11977. http://dx.doi.org/10.1039/c2dt31198d

7. Back, T. Curr. Green Chem. 2016, 3, 76-91.

http://dx.doi.org/10.2174/2213346103666160127003954

8. Pacuła, A. J.; Mangiavacchi, F.; Sancineto, L.; Lenardão, E. J.; Ścianowski, J.; Santi, C. Curr. Chem. Biol. 2015, 9, 97-112 and references therein.

http://dx.doi.org/10.2174/2212796810666160120220725

9. Santi C. Organoselenium Chemistry: Between Synthesis and Biochemistry, Bentham Science Publishers, 2014.

10. Nogueira, C. W.; Rocha J. B. Arch. Toxicol. 2011, 85, 1313-1359 and references therein. http://dx.doi.org/10.1007/s00204-011-0720-3

11. Wirth, T. Angew. Chem. Int. Ed. 2015, 54, 10074-10076 and references therein. http://dx.doi.org/10.1002/anie.201505056

12. Fernandes, A. P.; Gandin, V. Biochim. Biophys. Acta. 2015, 1850, 1642-1660 and references therein. http://dx.doi.org/10.1016/j.bbagen.2014.10.008

13. Azad, G. K.; Tomar,. R. S. Mol. Biol. Rep. 2014, 41, 4865-4879 and references therein. http://dx.doi.org/10.1007/s11033-014-3417-x

14. Soriano-Garcia, M. Curr. Med. Chem. 2004, 11, 1657-1669 and references therein. http://dx.doi.org/10.2174/0929867043365053

15. Mugesh, G; du Mont, W. W; Sies, H. Chem. Rev. 2001, 101, 2125-2179 and references therein. http://dx.doi.org/10.1021/cr000426w

16. Granda, J. M.; Piekielska, K.; Wąsińska, M.; Kawecka, N.; Giurg, M. Synthesis 2015, 47, 3321-3332. http://dx.doi.org/10.1055/s-0034-1381011

17. Denmark, S. E; Chi, H. M. J. Am. Chem. Soc. 2014, 136, 8915-8918. http://dx.doi.org/10.1021/ja5046296

18. Kawamata, Y.; Hashimoto, T.; Maruoka, K. J. Am. Chem. Soc. 2016, 138, 5206-5209. http://dx.doi.org/10.1021/jacs.6b01462

19. Sancineto, L.; Tidei, C.; Bagnoli, L.; Marini, F.; Lenardão, E.J.; Santi, C. Molecules 2015, 20, 10496-10510. http://dx.doi.org/10.3390/molecules200610496

20. He, J.; Li, D.; Xiong, K.; Ge, Y.; Jin, H.; Zhang, G.; Hong, M.; Tian, Y.; Yin, J.; Zeng, H. Bioorg. Med. Chem. 2012, 20, 3816-3827.

http://dx.doi.org/10.1016/i.bmc.2012.04.033

21. Nascimento, V.; Ferreira, N. L.; Canto, R. F.; Schott, K. L.; Waczuk, E. P.; Sancineto, L.; Santi, C.; Rocha, J. B.; Braga, A. L. Eur. J. Med. Chem. 2014, 87, 131-139.

http://dx.doi.org/10.1016/j.ejmech.2014.09.022

22. Thenin-Houssier, S.; de Vera, I.M.; Pedro-Rosa, L.; Brady, A.; Richard, A.; Konnick, B.; Opp, S.; Buffone, C.; Fuhrmann, J.; Kota, S.; Billack, B.; Pietka-Ottlik, M.; Tellinghuisen, T.; Choe, H.; Spicer, T.; Scampavia, L.; Diaz-Griffero, F.; Kojetin, D. J.; Valente, S. T. Antimicrob. Agents Chemother. 2016, 60, 2195-2208. http://dx.doi.org/10.1128/AAC.02574-15

23. Sancineto, L.; Mariotti, A.; Bagnoli, L.; Marini, F.; Desantis, J.; Iraci, N.; Santi, C.; Pannecouque, C.; Tabarrini, O. J. Med. Chem. 2015, 58, 9601-9614. http://dx.doi.org/10.1021/acs.jmedchem.5b01183 
24. Wójtowicz, H.; Chojnacka, M.; Młochowski, J.; Palus, J.; Syper, L.; Hudecova, D.; Uher, M.; Piasecki, E. ; Rybka, M. Farmaco 2003, 58, 1235-1242. http://dx.doi.org/10.1016/j.farmac.2003.08.003

25. Wójtowicz, H.; Kloc, K.; Maliszewska, I.; Młochowski, J.; Piętka, M.; Piasecki, E. Farmaco 2004, 59, 863868.

http://dx.doi.org/10.1016/i.farmac.2004.07.003

26. Piętka-Ottlik, M.; Wójtowicz-Młochowska, H.; Kołodziejczyk, K.; Piasecki, E.; Młochowski, J. Chem. Pharm. Bull. 2008, 56, 1423-1427.

http://dx.doi.org/10.1248/cpb.56.1423

27. Chan, G.; Hardej, D.; Santoro, M.; Lau-Cam, C.; Billack, B. J. Biochem. Mol. Toxicol. 2007, 21, $252-264$. http://dx.doi.org/10.1002/jbt.20189

28. Kamigata, N.; lizuka, H.; Izuoka, A.; Kobayashi M. Bull. Chem. Soc. Jpn. 1986, 59, $2179-2183$. http://dx.doi.org/10.1246/bcsj.59.2179

29. Mlochowski, J.; Gryglewski, R. J.; Inglot, A. D.; Jakubowski, A.; Juchniewicz, L.; Kloc, K. Liebigs Ann. 1996, 1751-1755.

http://dx.doi.org/10.1002/jlac.199619961108

30. Młochowski, J.; Kloc, K.; Syper, L.; Inglot, A. D.; Piasecki, E. Liebigs Ann. Chem. 1993, $1239-1244$. http://dx.doi.org/10.1002/jlac.1993199301201

31. Giurg, M.; Syper, L. Phosphorus Sulfur Silicon Relat. Elem. 2008, 183, $970-985$. http://dx.doi.org/10.1080/10426500801900956

32. Küppers, J.; Schulz-Fincke, A. C.; Palus, J.; Giurg, M.; Skarżewski, J.; Gütschow, M. Pharmaceuticals 2016, 9, 43.

http://dx.doi.org/10.3390/ph9030043

33. Chang, T. C.; Huang, M. L.; Hsu, W.L.; Hwang, J. M.; Hsu, L. Y. Chem. Pharm. Bull. 2003, 51, $1413-1416$. http://dx.doi.org/10.1248/cpb.51.1413

34. Welter, A.; Christiaens, L.; Wirtz-Peitz Eur. Pat. Appl. 44 453, 1982; Chem. Abstr. 1982, 96, 199699.

35. Welter, A.; Fischer, H.; Christiaens, L.; Wendel, A.; Etschenberg, E. Ger. Offen. 3513 070, 1986. 